\title{
A functional Variant (Rs35592567) in TP63 at $3 q 28$ is Associated with Gastric Cancer Risk via Modifying its Regulation by MicroRNA-140
}

\author{
Chaomin Lua Yongwei Yang ${ }^{\mathrm{a}}$ Siyue Ma \\ aThe Department of General Surgery, The Tumor Hospital Affiliated to Zhengzhou University
}

\author{
Key Words \\ Rs35592567 • TP63 • Gastric cancer • MiR-140 • 3'-UTR
}

\begin{abstract}
Background/Aims: TP63 was believed to play an important role in the development of many malignancies, while the polymorphisms located at the miRNA binding sites within the $3^{\prime} U T R$ of TP63 mRNA may interfere with its expression. In this study, we aimed to study the role of TP63 regulation in the tumorigenesis of gastric cancer (GC). Methods: Computational and luciferase analysis were used to search and confirm the target of miR-140. Real-time PCR, westernblot, MTT assay, and flow cytometry cell cycle analysis were utilized to explore the molecular pathway of miR-140 involved in the progression of GC. Results: TP63 was identified as a direct target gene of miR-140. In HT-29 cells over-expressing miR-140, the luciferase activity was decreased when the cells were transfected with wild-type TP63 3'UTR, but remained unchanged when the cells were transfected with mutant 1 and mutant 2 TP63 3'UTR. In addition, the level of TP63 in HT-29 cells transfected with miR-140 mimic was evidently down-regulated, whereas the level of TP63 in HT-29 cells transfected with miR-140 inhibitor was significantly up-regulated. Furthermore, based on the results from MTT assay and flow cytometry cell cycle analysis, HT-29 cells transfected with miR-140 mimics were associated with significantly higher viability compared to the cells transfected with the control plasmid, suggesting that an increased expression of miR-140 protected HT-29 cells against apoptosis. Finally, when miR-140 expression was high, the number of cells at the G1 phase was notably increased, accompanied by a remarkably diminished number of cells at the $\mathrm{S}$ phase. Conclusions: The rs35592567 polymorphism in TP63 affected the expression of TP63 by interfering with its interaction with miR-140, and could serve as an explanation for the increased risk of GC.

\section{Introduction}

Gastric cancer (GC) is the third most frequently diagnosed cancer in the world and the second most deadly cancer in both males and females [1]. GC greatly affects public health and

Chaomin Lu

KARGER
The Department of General Surgery, The Tumor Hospital Affiliated to Zhengzhou University, 127 Dongming Rd, Zhengzhou, 450000 (China)

E-Mail rs35592567@yeah.net 
caused about 231, 193 deaths in China in 2008 [2]. The prognosis of GC remains poor even though significant progresses have been made in the treatment of GC. A majority of GC cases are diagnosed in their late stage and the five-year survival rate remains at only $20 \%-30 \%$ [3]. A study has shown that squamous dysplasia and carcinoma may be originated from the basal cells of the esophageal squamous epithelium, and that p63 may be critical in esophagus squamous carcinogenesis [4]. By assaying the expression of p63 in keratinocyte cultures and murine tissues, some studies showed that p63 functions as a marker of precursor cells and may play a regulatory role in the epithelial proliferation and differentiation of cancer cells $[5$, 6]. Such hypothesis is also supported by the severe derangements of epithelial development in mice harboring a mutation of the p63 gene [5].

As single stranded and evolutionarily conserved non-coding RNAs, microRNAs (miRNA) have been shown to modulate the expression of their target genes and thus are implicated in a variety of biological processes, such as stem cell development, cell differentiation, proliferation, apoptosis, and tumorigenesis [7].

Single-nucleotide polymorphisms (SNPs) are the most common type ( $90 \%)$ of variants in the human genome. It is clear that miRNAs can bind to the cis-regulatory regions in the $3^{\prime}$-UTRs of their target genes to modulate gene expression. The complementarity between a miRNA and the corresponding region in its target mRNA(s) can be changed by a nucleotide variant in the 3' UTR sequences, hence impacting the affinity of miRNA binding. Therefore, these 3' UTR-SNPs may lead to variations in the expression of their target genes [8].

Abnormal miRNA expression was found in the development and progression of different cancers, such as esophageal cancer and GC $[9,10]$. GC is caused by multiple factors, including the interaction between environmental factors and multiple genes. On the other hand, miRNAs are main regulatory factors of the epigenome and are related to the alterations in many disease phenotypes [11]. Thereby, an early GC diagnosis and classification as well as a better GC prognosis may be achieved by identifying the miRNAs involved in the tumorigenesis of GC. For example, 9 miRNAs have been demonstrated to affect the overall survival of GC. In addition, it has been shown that a single nucleotide polymorphism (SNP) (rs35592567) in the miR-140 binding site within the 3'UTR of TP63 led to an elevated risk of bladder cancer $[12,13]$. Therefore, it is hypothesized in this study that rs35592567 may affect the interplay between miR-140 and TP63 mRNA , and the minor allele of rs35592567 may increase the risk of GC.

\section{Materials and Methods}

Sample collection

Peripheral blood samples from two groups of bone fracture patients were collected post their surgery, using a Lymphocyte Separation Medium (Human) (Applygen Technologies Inc. Beijing, China). A total of 1, 150 volunteers were enrolled in our research, which consisted of 621 GC patients and 529 healthy subjects. The demographic and clinicopathological characteristics of these participants, such as age, sex, tumor stage and tumor grade, were summarized in Table 1. All procedures of this study were approved by the Ethics Committee at our institute and performed according to the Declaration of Helsinki. All participants of this study signed the form of informed consent.

\section{Genotyping Taqman assay}

A QIAmp Kit (Qiagen, Valencia, CA, USA) was utilized to isolate genomic DNA from the peripheral blood samples collected from the subjects. A PCR-based direct DNA sequence analysis was used to locate the SNP (rs35592567) using an ABI 7900HT Real-Time PCR System and a TaqMan SNP Genotyping assay (Applied Biosystems, Foster City, CA, USA).

RNA isolation and real-time PCR

A RNeasy total RNA isolation system (QIAGEN, Valentia, CA, USA) was utilized to extract the total RNA from cultured cells. A RNA PCR kit containing avian myeloblastosis virus RTase (Takara, Tokyo, Japan) was

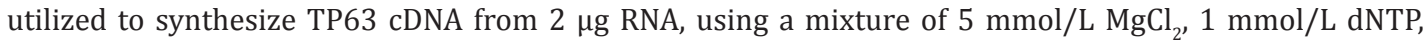




\begin{tabular}{|c|c|c|}
\hline Cellular Physiology & Cell Physiol Biochem 2018; & 47:235-244 \\
\hline and Biochemistry & $\begin{array}{l}\text { DOI: 10.1159/000489802, } \\
\text { Published online: May I6, } 2018\end{array}$ & $\begin{array}{l}\text { O } 2018 \text { The Author(s). Published by S. Karger AG, Basel } \\
\text { www.karger.com/cpb }\end{array}$ \\
\hline
\end{tabular}

Lu/Yang/Ma: Rs35592567 is Associated with Gastric Cancer Risk

$0.25 \mathrm{U} / \mu \mathrm{L}$ reverse transcriptase, and a single-strength RNA PCR buffer containing $50 \mathrm{mmol} / \mathrm{L}$ $\mathrm{KCl}, 10 \mathrm{mmol} / \mathrm{L}$ Tris-HCl, and $2.5 \mu \mathrm{mol} / \mathrm{L}$ oligo dT primer. Before PCR amplification, the above mixture was incubated for $40 \mathrm{~min}$ at $42^{\circ} \mathrm{C}$, and then heated for $5 \mathrm{~min}$ at $99^{\circ} \mathrm{C}$ before being chilled on ice. A thermal cycler (Perkin-Elmer, Hayward, CA, USA) was utilized to amplify the TP63 cDNA using $80 \mu \mathrm{L}$ of the mixture containing the single-strength RNA PCR buffer, $2.5 \mathrm{U}$ of Taq DNA polymerase, 4 $\mathrm{mmol} / \mathrm{L} \mathrm{MgCl}_{2}$, and $0.2 \mu \mathrm{mol} / \mathrm{L}$ of each primer. The conditions of the thermal cycle was set as follows: $15 \mathrm{~min}$ at $95^{\circ} \mathrm{C}$ for initial activation, followed by $60 \mathrm{~s}$ at $94^{\circ} \mathrm{C}$ for denaturation, $60 \mathrm{~s}$ at $53^{\circ} \mathrm{C}$ for annealing, and $120 \mathrm{~s}$ at $72^{\circ} \mathrm{C}$ for extension. An $\mathrm{ABI}$ 7900HT Sequence Detection System (Applied Biosystems, Foster City, CA) was used in conjunction with SYBR-Green I (Qiagen, Hilden, Germany) to perform real-time PCR and measure the expression of miR-140 and TP63. 1.5\% agarose gel electrophoresis was utilized to separate PCR products. The NIH Image software (National Institutes of Health; Bethesda, MD, USA) was utilized to perform densitometric scanning and to calculate the expression of target genes. GAPDH and U6 were served as internal control. All experiments were carried out in triplicates.

\section{Cell culture and transfection}

RPMI containing 10\% heat-inactivated FBS (fetal bovine serum), $50 \mathrm{U} / \mathrm{mL}$ streptomycin, $50 \mathrm{U} / \mathrm{mL}$ penicillin and $2 \mathrm{mM}$ glutamine (Euroclone, UK) was used to culture $\mathrm{HGC}-27$ and BGC-823 cells at $37^{\circ} \mathrm{Cin}$ a humidified atmosphere containing $5 \% \mathrm{CO}_{2} / 95 \%$ air. Prior to transfection, the cells were grown to 10,000 cells $/ \mathrm{cm}^{2}$ and were transfected with miR-140 mimic or inhibitor (Life Technologies, Italy), TP63 siRNA, or a scramble control using the Attractene Transfection Reagent (Qiagen, Hilden, Germany). All tests were carried out in triplicates.

\section{Cell proliferation assay}

The viability of HGC-27 and BGC-823 cells was examined using a modified MTT (3-[4, 5-dimethylthiazol-2-yl)-2, 5-diphenyltetrazolium bromide) assay. In brief, cultured cells were transfected with miR140 mimic or inhibitor, and returned into the incubator for 48 hours of additional culture. Subsequently, $10 \mu \mathrm{L}$ MTT AB solution (Millipore, Billerica, MA, USA) were added into each well, followed by 4 hours of additional incubation. An ELISA reader (ELX-800 type, Bio-Tek, Vermont, USA) was utilized to measure the optical density of the cells at $570 \mathrm{~nm}$ and to quantify cell proliferation. Each experiment was repeated three times.

\section{Luciferase assay}

The full fragment of TP63 3'UTR containing the potential binding site of miR-140 was amplified using PCR and the binding site was mutated using a Quik-Change Site-Directed Mutagenesis Kit (Stratagene, LaJolla, CA, USA). Subsequently, wild-type and mutant TP63 3'UTR was inserted into a pGL3-control vector (Promega, Madison, WI) immediately downstream of the luciferase reporter gene to generate different 


\section{Cellular Physiology Cell Physiol Biochem 2018;47:235-244 \begin{tabular}{l|l} 
DOI: 10.1159/000489802 & Ond Biochemistry \\
Published online: Nay 16, 2018 & $\begin{array}{l}\text { 2018 The Author(s). Published by S. Karger AG, Basel } \\
\text { www.karger.com/cpb }\end{array}$
\end{tabular}}

Lu/Yang/Ma: Rs35592567 is Associated with Gastric Cancer Risk

luciferase constructs. The cells were plated into 96-well plates at a final density of $1 \times 10^{5}$ cells/well and cultured for 12 hours, followed by co-transfection with $0.5 \mathrm{mg}$ pGL3 constructs, $0.07 \mathrm{mg}$ pRL-CMV plasmids (Promega, Madison, WI), and a miR-140 mimic or inhibitor. The transfection was carried out using Lipofectamine 3000 (Invitrogen, CA, US). At 48 hours post-transfection, a Dual Luciferase Reporter Assay System (Promega, Madison, WI) was utilized to measure the activity of firefly and renilla luciferase. The expression of different constructs was calculated by normalizing the firefly signal to the activity of renilla luciferase. Each test was repeated three times.

\section{Western blot analysis}

A RIPA buffer (Thermo Scientific, Waltham, MA, USA) was used to lyse the cells at 48 hour after transfection. A BCA assay (Thermo Scientific, Waltham, MA, USA) was utilized to measure the protein concentration. The lysates were re-suspended in a loading buffer and denatured for $10 \mathrm{~min}$ at $100^{\circ} \mathrm{C} .10$ $\%$ SDS-PAGE was utilized to separate $20 \mu \mathrm{g}$ proteins, followed by electric transferring of the proteins onto PVDF membranes at $90 \mathrm{~V}$ for $120 \mathrm{~min}$. A TBST buffer $(0.1 \%$ Tween-20) containing $5 \%$ fat free milk was utilized to block the membranes at room temperature for $120 \mathrm{~min}$. Subsequently, the membranes were incubated in anti- $\beta$-actin antibody (1:8000, Sigma, St. Louis, MO, USA) and rabbit polyclonal anti-TP63 antibody (1:5000, Abcam, Cambridge, UK) for 12 hours at $4^{\circ} \mathrm{C}$. The protein bands were visualized using horseradish peroxidase (HRP)-labeled anti-rabbit secondary antibodies (1:15000, Sigma, St. Louis, MO, USA). The auto radiographs were quantified using a Chemiluminescence assay (Amersham Pharmacia Inc, Piscataway, NJ, USA) . Each independent test was carried out three times.

\section{Cell apoptosis}

The apoptosis of cultured cells was evaluated using a FITC Annexin V Apoptosis Detection Kit and PI (Biolegend, San Diego, CA). At 48 hours after the cultured cell were transfected with a miR-140 mimic or inhibitor, the cells were harvested and washed twice in a cold BioLegend's Cell Staining Buffer (Biolegend, San Diego, CA). Subsequently, the cells were resuspended in an Annexin V Binding Buffer (Biolegend, San Diego, CA) at a final density of $1 \sim 2 \times 10^{5}$ cells per well. The cells were then stained in $5 \mu \mathrm{L}$ of FITC Annexin $\mathrm{V}$ and $10 \mu \mathrm{L}$ of propidium iodide (Biolegend, San Diego, CA) for $15 \mathrm{~min}$ in dark and at room temperature. Finally, the cells were suspended in $400 \mu \mathrm{L}$ of Annexin V Binding Buffer (Biolegend, San Diego, CA) and analyzed by flow cytometry. Each experiment was repeated three times.

\section{Cell cycle analysis}

The cultured cells were trypsinized using a Cycle TESTPLUS DNA reagent kit (BD Biosciences, San Jose, CA, USA) and stained in propidium iodide. A FACS Calibur (BD Biosciences, San Diego, CA) and FlowJo 7.6 software (Tree Star, Ashland, OR, USA) were utilized to analyze the stained cells. Each independent test was repeated three times.

\section{Statistical analysis}

All experiments were carried out three times and all data were shown as mean \pm SD (standard deviation). Prism 4 (GraphPad Software Inc., CA, USA) was used to perform all statistical analyses. ANOVA (two-way analysis of variance), student t-test and Bonferroni's or Dunnett's multiple comparison test were utilized to compare the results. A P values of $<0.05$ was considered statistically significant.

\section{Results}

Demographic, clinicopathological and genotypic parameters of the participants recruited in this study

SPSS version 16.0 software and student $t$ test were used to analyze the demographic and clinicopathological characteristics among the participants from the two groups, and no significant difference was observed with respect to age, sex, tumor stage and tumor grade. Using a logistic regression analysis based on a recessive genetic model rather than a dominant model, it was found that the rs35592567 polymorphism in the TT genotype was closely associated with an increased risk of GC, as shown in Table 2. 
TP63 may act as a direct target of $\mathrm{miR}-140$

Subsequently, we sought to evaluate a potential target not previously validated for miR-140. Prediction algorithms, including the UCSC Genome browser (http://www.genome.ucsc. edu), were utilized to predict the target gene of miR-140. As shown in Fig. 1, TP63 was identified as a putative target for miR-140, with a complimentary binding site located in the 3'UTR of TP63. Subsequently, the 8bp putative target site (seed sequence) in TP63 was mutated and named Mutant 1 TP63, followed by a C-to-T substitution carried out at one base of this 8bp putative target site, with the product named as Mutant 2 TP63.

TP63 was confirmed as a direct target of $m i R-140$

In the next step, reporter constructs containing wild-type TP63, Mutant 1 TP63 or Mutant 2 TP63 were co-transfected into HGC-27 and BGC-823 cells with miR-140, and the relative luciferase activity in these cells was measured. As shown in Fig. 2, in both HGC-27 (Fig. 2A) and BGC-823 (Fig. 2B) cells, the luciferase activity of wild-type TP63 in cells over-expressing miR-140 was significantly decreased as compared with the negative control (NC), whereas the luciferase activities of Mutant 1 TP63 or Mutant 2 TP63 in cells over-expressing miR-140 displayed no apparent change, indicating that TP63 acted as a direct target of miR-140 and the binding of miR-140 to TP63 was dependent on the $\mathrm{C}$ allele located in the $8 \mathrm{bp}$ sequences.

\section{MiR-140 downregulated endogenous TP63 expression in HGC-27 and BGC-823 cells}

To further test whether miR-140 could negatively regulate TP63 expression in HGC-27 and BGC-823 cells, the effect of miR-140 expression on the endogenous level of TP63 mRNA and protein was examined in HGC-27 and BGC-823 cells transfected with an miR-140 mimic or inhibitor. As shown in Figs. 3 and 4, the over-expression of miR-140 in HGC-27 (Fig. 3) and BGC-823 (Fig. 4) cells dramatically inhibited the expression of both TP63 mRNA (Figs. 3A and 4A) and protein (Figs. 3B and 4B). Similarly, the downregulation of miR-140 in HGC-27 (Fig. 3) and BGC-823 (Fig. 4) cells significantly promoted the expression of both TP63 mRNA (Figs. 3A and 4A) and protein (Figs. 3B and 4B), indicating that miR-140 directly suppressed the expression of TP63 in both HGC-27 and BGC-823 cells.

Functions of miR-140 in HGC-27 and BGC-823 cells

Subsequently, the impact of miR-140 on cell viability, apoptosis and cell cycle was evaluated by transfecting HGC-27 and BGC-823 cells with either a miR-140 mimic, a miR-140 


\section{Cellular Physiology \begin{tabular}{l|l} 
and Biochemistry Published 10.1159/000489802 & $\begin{array}{l}\text { (C) } 2018 \text { The Author(s). Published by S. Karger AG, Basel } \\
\text { www.karger.com/cpb }\end{array}$
\end{tabular} \\ Lu/Yang/Ma: Rs35592567 is Associated with Gastric Cancer Risk}

Fig. 2. HGC-27 and BGC-823 cells transfected with negative control or miR-140 and luciferase reporters either carried wild-type TP63 or Mutant 1 TP63 or Mutant 2 TP63. A: MiR-140 decreased luciferase activity of wild-type TP63, but not those of mutant1 and mutant2 TP63 in HGC-27 cell. B: MiR-140 decreased luciferase activity of wild-type TP63, but not those of mutant 1 and mutant2 TP63 in BGC-823 cell.

inhibitor or NC. Cell viability was detected using MTT assays, while cell apoptosis was examined using an Annexin V-FITC apoptosis detection kit in conjunction with flow cytometry (FCM). Cell cycle was assayed with flow cytometry. As shown in Figs. $5 \mathrm{~A}$ and $6 \mathrm{~A}$, from 12 hours to 72 hours post transfection, HGC-27 (Fig. 5A) and BGC-823 cells (Fig. 6A) transfected with miR-140 mimics showed significantly increased cell viability in a time-dependent manner, whereas HGC-27 cells transfected with miR-140 inhibitors exhibited evidently lower cell viability over time. As shown in Figs. 5B and 6B, an increased expression of miR-140 protected HGC-27 (Fig. 5B) and BGC-823 cells (Fig. 6B) against apoptosis, whereas a decreased expression of miR140 promoted the apoptosis of HGC-27 (Fig. 5B) and BGC-823 cells (Fig. 6B). As shown in Figs. 5C and 6C, the number of HGC-27 (Fig. 5C) and BGC-823 cells (Fig. 6C) at the G1 phase was notably increased upon miR140 over-expression, accompanied by a significantly decreased number of HGC-27 (Fig. 5C) and BGC-823 cells (Fig. 6C) at the S phase. All these results suggested that miR140 inhibited cell proliferation by arresting HGC-27 and BGC-823 cells at the G1 phase, thus enhancing the apoptosis of HGC-27 and BGC-823 cells.

\section{Discussion}

The expression characteristic of miR-140-5p in normal and tumor tissues suggested its potential role of tumorinhibition in many cancers [14]. Zhai et al. showed that the tumor formation and metastasis ability of colon cancer stem cells can be abolished by the overexpression of miR-140-5p, which
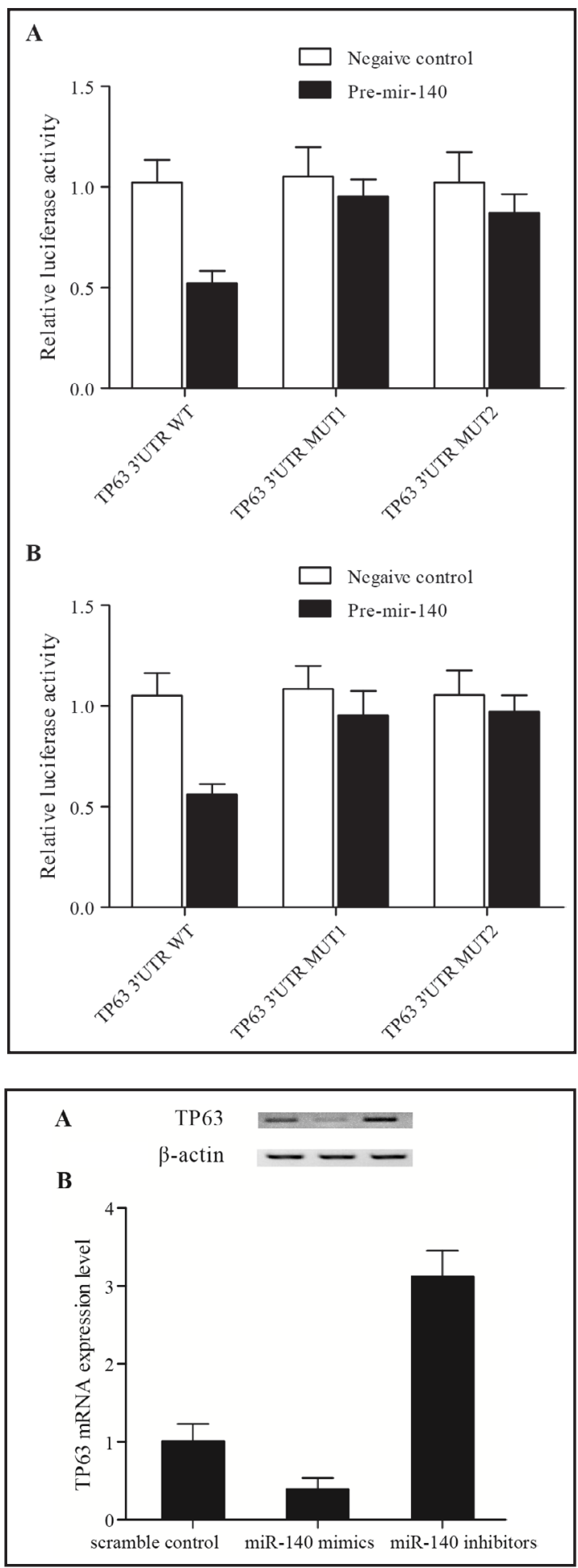

Fig. 3. The TP63 expression levels of HGC-27 cells transfected with miR-140 mimic or miR-140 inhibitor were examined. A: MiR-140 mimic reduced TP63 protein level, and miR-140 inhibitor induced TP63 protein level. B: MiR-140 mimic reduced TP63 mRNA level, and miR-140 inhibitor induced TP63 mRNA level. 
Fig. 4. The TP63 expression levels of BGC-823 cells transfected with miR-140 mimic or miR-140 inhibitor were examined. A: MiR-140 mimic reduced TP63 protein level, and miR-140 inhibitor induced TP63 protein level. B: MiR-140 mimic reduced TP63 mRNA level, and miR-140 inhibitor induced TP63 mRNA level.

directly affected a target known as Smad2 [15]. In addition, primary tumor tissues showed a progressive loss in miR-140-5p expression as compared with that in normal colorectal mucosa, whereas tissues affected by liver metastasis were associated with a further decreased expression of miR140-5p. Furthermore, an elevated miR140-5p expression was found to be closely related to a better prognosis in stage III and IV CRC patients. In contrast, Mosakhani et al. discovered that the upregulation of miR-140-5p was closely associated with worse overall survival in metastatic CRC patients harboring a wild type KRAS/BRAF [15]. In addition, downregulated miR-140-5p expression was closely related to poorer overall survival, an advanced clinical stage and lymph node metastasis [16].

It has been shown that miR-140$5 \mathrm{p}$ functions as a tumor suppressor in hepatocellular carcinoma, tongue squamous cell carcinoma, and nonsmall cell lung cancer [17-19]. For example, in lung cancer, cancer cell proliferation was suppressed by miR140-5p that targeted MMD and inhibited the activation of the MAPK signaling pathway [20]. Furthermore, miR-140-5p alleviates the metastasis and progression of tongue squamous cell carcinoma by down-regulating the expression of PAX6, HDAC7 and LAMC1 [21]. MiR-140-5p also inhibited the proliferation and metastasis of HCC by suppressing the MAPK signaling pathway [22], while the overexpression of miR-140-5p triggered the apoptosis of ovarian cancer cells and substantially suppressed their proliferation [23]. In this study, a computational analysis was performed to identify TP63 as a putative target of miR-140. Subsequent luciferase assay in HGC-27 and BGC-823 cells confirmed that TP63 acted as a direct
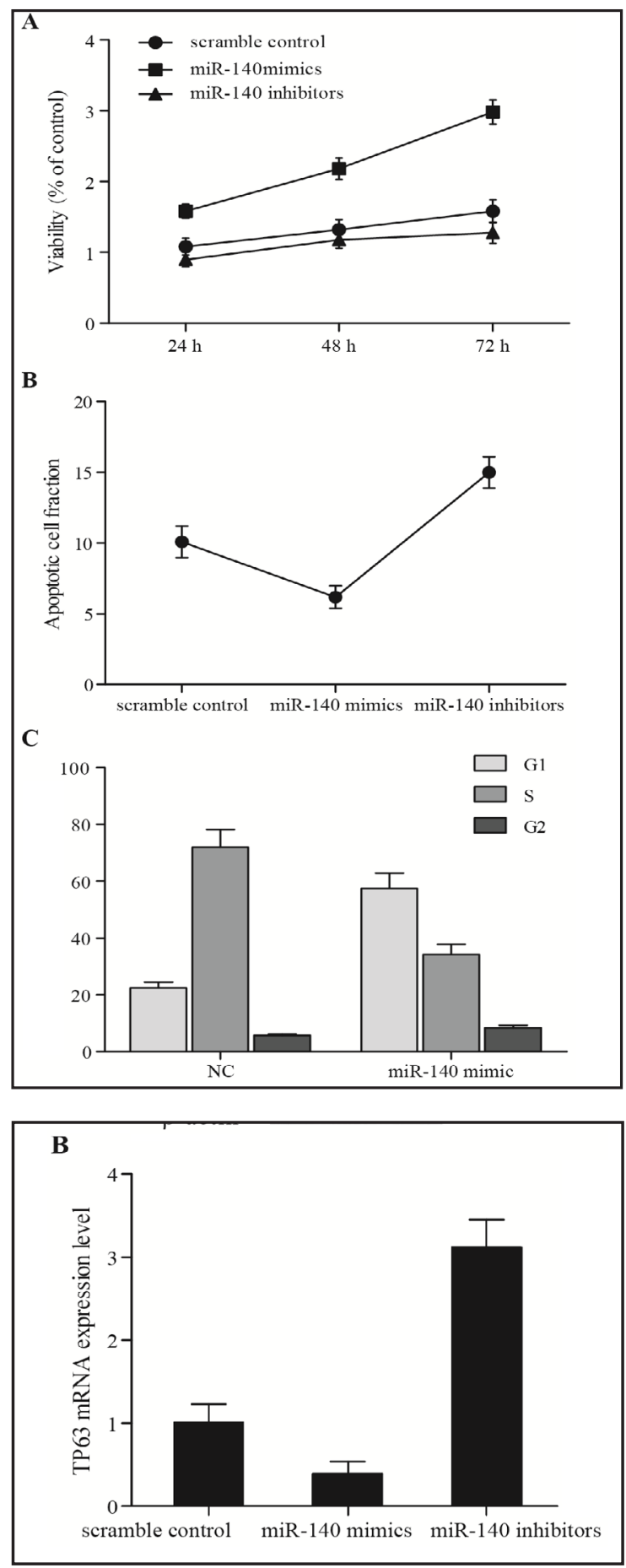

Fig. 5. The impact of miR-140 on cell survival, cell apoptosis and cell cycle were determined following introduction with miR-140 mimic or inhibitor in HGC27 cell. A: MiR-140 mimic obviously and time-dependently promoted cell viability, while miR-140 inhibitor inhibited proliferation of cell. B: MiR-140 mimic strikingly suppressed cell apoptosis, and miR-140 inhibitor evidently provoked apoptosis. C: MiR-140 mimic arrest HGC-27 cell at G1 phase. 
target of miR-140. Furthermore, realtime PCR and western-blot analysis were performed to explore the relationship between miR-140 and TP63 in HGC27 and BGC-823 cells, and the results revealed that the up-regulation of miR140 expression in HGC-27 and BGC-823 cells dramatically inhibited the expression of both TP63 mRNA and protein.

As a member of the p53 family, p63 is encoded by the TP63 gene on chromosome 3q27. Similar to TP53 and TP73, TP63 encodes a sequencespecific regulatory protein, whose transcription leads to the activation of target genes involved in a variety of cellular pathways. However, unlike the p53 protein, p63 protein is not associated with tumor inhibition, yet plays a key role as a regulatory protein in craniofacial, epithelial and limb development [24]. So far, at least six different isoforms of p63 have been discovered. Three of these isoforms contain a transactivation domain (TAp63), whereas the other three isoforms do not contain $(\Delta \mathrm{Np} 63)[25]$.

Asioli et al. demonstrated that p63 could potentially be used as a prognostic marker for Merkel cell carcinoma [25]. Recently, Hall et al. have shown that p63 expression is a risk factor closely related to a reduced rate of survival [26]. In another study involving 17 cases, an association between p63 expression and unfavorable prognosis in Merkel cell carcinoma (MCC) was revealed [27]. On the contrary, Lim et al. suggested that p63 expression was not significantly correlated with disease

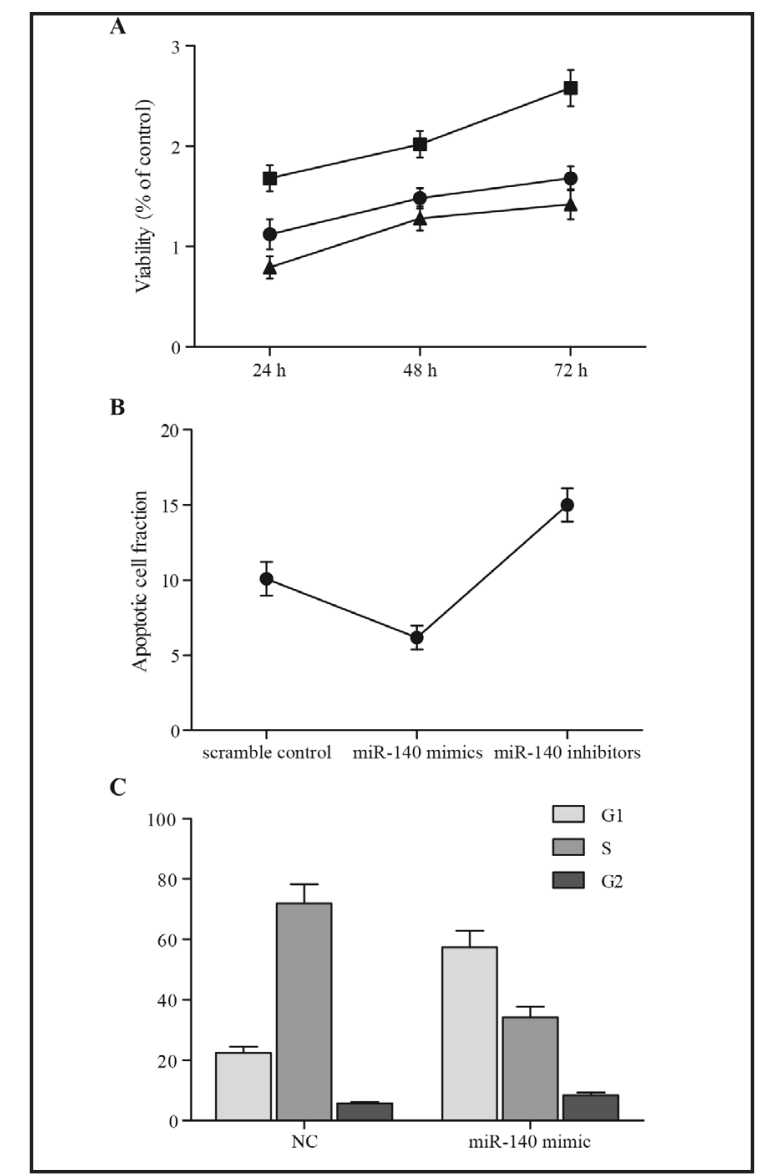

Fig. 6. The impact of miR-140 on cell survival, cell apoptosis and cell cycle were determined following introduction with miR-140 mimic or inhibitor in BGC-823 cell. A: MiR-140 mimic obviously and timedependently promoted cell viability, while miR-140 inhibitor inhibited proliferation of cell. B: MiR-140 mimic strikingly suppressed cell apoptosis, and miR140 inhibitor evidently provoked apoptosis. C: MiR140 mimic arrest HGC-27 cell at G1 phase. outcome, since positive p63 expression could only be observed in $9 \%$ of the patients [28]. The role of p63 as a prognostic factor has been studied in MCC using a multivariate model, which predicted that the p63 expression in MCC tumor cells was closely related to worse disease-specific survival and increased the mortality of MCC by approximately two-fold [29]. In this study, the effect of miR-140 on cell survival, cell apoptosis and cell cycle was investigated using MTT assay and cell cycle analysis, respectively, and it was found that the transfection of miR-140 mimics significantly increased the viability of HGC-27 and BGC-823 cells in a time-dependent manner. In contrary, the transfection of miR-140 inhibitor remarkably decreased the viability of HGC-27 and BGC-823 cells, also in a time-dependent manner. In addition, the number of HGC-27 and BGC-823 cells arrested at the G1 phase was much higher upon overexpression of miR-140, accompanied by a dramatically reduced number of cells at the $S$ phase.

An earlier report has shown that gene expression could be impacted by SNPs situated in the miRNA binding site of its target genes [30]. The sequencing and bioinformatics-based analyses have predicted the location of SNP rs35592567 within a hypothetical binding site for miR-140-5p. Furthermore, it has been shown that, by impacting the binding of miR-140- 
$5 p$ to the 3'-UTR of TP63, rs35592567 could lead to a reduced expression of TP63 [12]. It has also been that the rs35592567 SNP in the miR-140-5p binding site could reduce the expression of TP63 and reduce the risk of bladder cancer, thus explaining the role of a previous unidentified casual loci on 3q28 in GWAS of bladder cancer [12].

\section{Conclusion}

The findings of this study demonstrated that TP63 is a direct target of miR-140. The presence of rs35592567 polymorphism within the 3'UTR of TP63 altered the expression of TP63 by modulating its interaction with miR-140. Finally, the rs35592567 polymorphism is found to be associated with the risk of GC in the Chinese population.

\section{Disclosure Statement}

The authors declare to have no conflict of interests.

\section{References}

1 Chen XZ, Chen H, Castro FA, Hu JK, Brenner H: Epstein-Barr virus infection and gastric cancer: a systematic review. Medicine (Baltimore) 2015;94:e792. Qinghai Z, Yanying W, Yunfang C, Xukui Z, Xiaoqiao Z: Effect of interleukin-17A and interleukin-17F gene polymorphisms on the risk of gastric cancer in a Chinese population. Gene 2014;537:328-332. Tahara T, Shibata T, Nakamura M, Yamashita H, Yoshioka D, Okubo M, Yonemura J, Maeda Y, Maruyama N, Kamano T, Kamiya Y, Fujita H, Nakagawa Y, Nagasaka M, Iwata M, Hirata I, Arisawa T: Association between IL-17A, -17F and MIF polymorphisms predispose to CpG island hyper-methylation in gastric cancer. Int J Mol Med 2010;25:471-477.

Glickman JN, Yang A, Shahsafaei A, McKeon F, Odze RD: Expression of p53-related protein p63 in the gastrointestinal tract and in esophageal metaplastic and neoplastic disorders. Hum Pathol 2001;32:1157-1165. C, McKeon F: p63 is essential for regenerative proliferation in limb, craniofacial and epithelial development. Nature 1999;398:714-718. Pellegrini G, Dellambra E, Golisano 0, Martinelli E, Fantozzi I, Bondanza S, Ponzin D, McKeon F, De Luca M: p63 identifies keratinocyte stem cells. Proc Natl Acad Sci U S A 2001;98:3156-3161. Sassen S, Miska EA, Caldas C: MicroRNA: implications for cancer. Virchows Arch 2008;452:1-10. Kim J, Bartel DP: Allelic imbalance sequencing reveals that single-nucleotide polymorphisms frequently alter microRNA-directed repression. Nat Biotechnol 2009;27:472-477.

Xu X, Zhang Y, Liu Z, Zhang X, Jia J: miRNA-532-5p functions as an oncogenic microRNA in human gastric cancer by directly targeting RUNX3. J Cell Mol Med 2016;20:95-103. Qi B, Liu SG, Qin XG, Yao WJ, Lu JG, Guo L, Wang TY, Li HC, Zhao BS: Overregulation of microRNA-212 in the poor prognosis of esophageal cancer patients. Genet Mol Res 2014;13:7800-7807. Borel C, Antonarakis SE: Functional genetic variation of human miRNAs and phenotypic consequences. Mamm Genome 2008;19:503-509.

12 Wang M, Du M, Ma L, Chu H, Lv Q, Ye D, Guo J, Gu C, Xia G, Zhu Y, Ding Q, Yuan L, Fu G, Tong N, Qin C, Yin $\mathrm{C}, \mathrm{Xu}$ J, Zhang Z: A functional variant in TP63 at 3q28 associated with bladder cancer risk by creating an miR-140-5p binding site. Int J Cancer 2016;139:65-74.

13 Tannapfel A, Schmelzer S, Benicke M, Klimpfinger M, Kohlhaw K, Mossner J, Engeland K, Wittekind C: Expression of the p53 homologues p63 and p73 in multiple simultaneous gastric cancer. J Pathol 2001;195:163-170. 


\section{Cellular Physiology Cell Physiol Biochem 2018;47:235-244

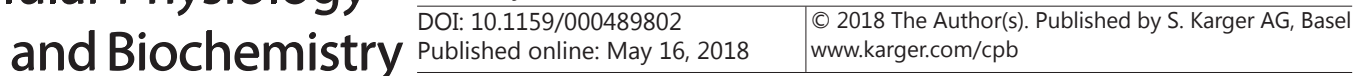

Lu/Yang/Ma: Rs35592567 is Associated with Gastric Cancer Risk

14 Song B, Wang Y, Xi Y, Kudo K, Bruheim S, Botchkina GI, Gavin E, Wan Y, Formentini A, Kornmann M, Fodstad 0, Ju J: Mechanism of chemoresistance mediated by miR-140 in human osteosarcoma and colon cancer cells. Oncogene 2009;28:4065-4074.

15 Zhai H, Fesler A, Ba Y, Wu S, Ju J: Inhibition of colorectal cancer stem cell survival and invasive potential by hsa-miR-140-5p mediated suppression of Smad2 and autophagy. Oncotarget 2015;6:19735-19746. Zhang W, Zou C, Pan L, Xu Y, Qi W, Ma G, Hou Y, Jiang P: MicroRNA-140-5p inhibits the progression of colorectal cancer by targeting VEGFA. Cell Physiol Biochem 2015;37:1123-1133. Hwang S, Park SK, Lee HY, Kim SW, Lee JS, Choi EK, You D, Kim CS, Suh N: miR-140-5p suppresses BMP2-mediated osteogenesis in undifferentiated human mesenchymal stem cells. FEBS Lett 2014;588:2957-2963.

Kai Y, Peng W, Ling W, Jiebing H, Zhuan B: Reciprocal effects between microRNA-140-5p and ADAM10 suppress migration and invasion of human tongue cancer cells. Biochem Biophys Res Commun 2014;448:308-314.

19 Li W, He F: Monocyte to macrophage differentiation-associated (MMD) targeted by miR-140-5p regulates tumor growth in non-small cell lung cancer. Biochem Biophys Res Commun 2014;450:844850.

Yang H, Fang F, Chang R, Yang L: MicroRNA-140-5p suppresses tumor growth and metastasis by targeting transforming growth factor beta receptor 1 and fibroblast growth factor 9 in hepatocellular carcinoma. Hepatology 2013;58:205-217. Shi Z, Luo G, Fu L, Fang Z, Wang X, Li X: miR-9 and miR-140-5p target FoxP2 and are regulated as a function of the social context of singing behavior in zebra finches. J Neurosci 2013;33:16510-16521. Karlsen TA, Jakobsen RB, Mikkelsen TS, Brinchmann JE: microRNA-140 targets RALA and regulates chondrogenic differentiation of human mesenchymal stem cells by translational enhancement of SOX 9 and ACAN. Stem Cells Dev 2014;23:290-304.

Lan H, Chen W, He G, Yang S: miR-140-5p inhibits ovarian cancer growth partially by repression of PDGFRA. Biomed Pharmacother 2015;75:117-122. cell carcinoma. Cancer 2007;110:640-647.

Hall BJ, Pincus LB, Yu SS, Oh DH, Wilson AR, McCalmont TH: Immunohistochemical prognostication of Merkel cell carcinoma: p63 expression but not polyomavirus status correlates with outcome. J Cutan Pathol 2012;39:911-917. Kim J, McNiff JM: Nuclear expression of survivin portends a poor prognosis in Merkel cell carcinoma. Mod Pathol 2008;21:764-769.

Lim CS, Whalley D, Haydu LE, Murali R, Tippett J, Thompson JF, Hruby G, Scolyer RA: Increasing tumor thickness is associated with recurrence and poorer survival in patients with Merkel cell carcinoma. Ann Surg Oncol 2012;19:3325-3334. ZB, Nghiem P: p63 expression in Merkel cell carcinoma predicts poorer survival yet may have limited clinical utility. Am J Clin Pathol 2013;140:838-844. Ryan BM, Robles AI, Harris CC: Genetic variation in microRNA networks: the implications for cancer research. Nat Rev Cancer 2010;10:389-402. 